University of Nebraska - Lincoln

DigitalCommons@University of Nebraska - Lincoln

Timothy J. Gay Publications

Research Papers in Physics and Astronomy

2011

Detecting an extended light source through a lens

E. T. Litaker

University of Nebraska-Lincoln, elitaker2@unl.edu

J. R. Machacek

University of Nebraska-Lincoln

Timothy J. Gay

University of Nebraska - Lincoln, tgay1@unl.edu

Follow this and additional works at: https://digitalcommons.unl.edu/physicsgay

Part of the Physics Commons

Litaker, E. T.; Machacek, J. R.; and Gay, Timothy J., "Detecting an extended light source through a lens" (2011). Timothy J. Gay Publications. 62.

https://digitalcommons.unl.edu/physicsgay/62

This Article is brought to you for free and open access by the Research Papers in Physics and Astronomy at DigitalCommons@University of Nebraska - Lincoln. It has been accepted for inclusion in Timothy J. Gay Publications by an authorized administrator of DigitalCommons@University of Nebraska - Lincoln. 


\title{
Detecting an extended light source through a lens
}

\author{
E T Litaker, J R Machacek ${ }^{1}$ and T J Gay \\ Department of Physics and Astronomy, University of Nebraska, Lincoln, NE 68588-0299, USA \\ E-mail: elitaker2@unl.edu
}

Received 7 April 2011, in final form 9 May 2011

Published 16 June 2011

Online at stacks.iop.org/EJP/32/1107

\begin{abstract}
We present a Monte Carlo simulation of a cylindrical luminescent volume and a typical lens-detector system. The results of this simulation yield a graphically simple picture of the regions within the cylindrical volume from which this system detects light. Because the cylindrical volume permits large angles of incidence, we use a modification of the thin-lens approximation for ray tracing. We compare simulation results with concepts from imaging optics, and comment on implications for experimental design.
\end{abstract}

\section{Introduction}

Optical systems of lenses in experimental apparatus are often used to collect light from extended fluorescent sources such as foil-excited atomic beams [1], electronically bombarded molecular gases [2], laser-induced fluorescence from a plasma [3], fluorescence generated by discharge from a plasma [4], or light sources found in teaching laboratories. When considering the placement of the lens-detector combination, a basic question naturally arises: what is the amount, extent and spatial distribution of what the system detects from within the source volume-in short, what does a lens-detector combination 'see'? The purpose of this paper is to provide a simple, graphical answer to this question (which does not appear to have been done previously in the literature), and to provide a beginning student designing his or her first optical system with an initial, qualitative reference point for what such systems can be expected to do.

Ray-tracing algorithms deal with image and object locations and help to quantify aberrations induced by lenses, but do not directly address collection of light from an extended source. The concepts of incoherent optical transfer functions [5], etendue [6] and isophotes [7] are related to these issues, but are either mathematically abstract and not easily accessible, or have not been used in the analysis of simple, axisymmetric optical systems designed to collect light. In this paper, we present a Monte Carlo analysis of a typical lens-detector system that

1 Present address: Australian National University, Canberra, ACT 2601, Australia. 


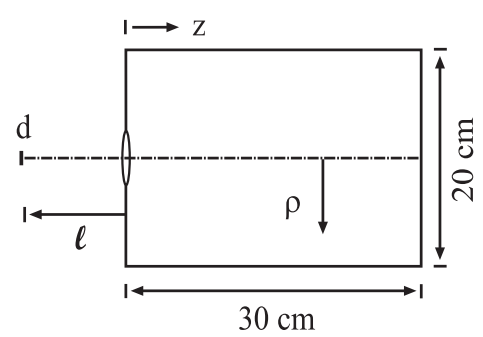

Figure 1. Lens/detector geometry (see the text). The lens has a focal length $10 \mathrm{~cm}$, and the cylindrical luminescent volume has a radius $10 \mathrm{~cm}$ and length $30 \mathrm{~cm}$. The lens is $5 \mathrm{~cm}$ in diameter, the detector is $1 \mathrm{~cm}$ in diameter, and the varied parameter in the simulation is $\ell$.

yields a graphically simple picture of how it collects light. We use the results of this analysis to comment on implications for experimental design.

\section{Background}

Consider a lens and its associated object and image spaces. The usual rudimentary analysis of an object and its image formed by a lens involves ray tracing and provides the location, size and orientation of the image. In addition to transverse magnification, it is also possible to determine longitudinal magnification [8]. Our goal is different. For a given detector distance from the lens, we wish to know how much light can be detected from within the volume, how much of the luminescent volume can be detected and the spatial distribution of what is detected.

We use ray tracing to analyse a prototypical optical system comprising a lens and a detector (figure 1). Since the range of the angles of incidence on the lens extends beyond the limits of the paraxial approximation, we use a modified ray-tracing approximation for our Monte Carlo simulation (see the appendix). In this approximation, Snell's law is used at each (biconvex) lens surface for arbitrary angles of incidence. For computational simplicity, we take the optical path length within the lens to be zero. This leads to significant deviation from true optical paths for rays with incident angles greater than $60^{\circ}$ which intersect the lens within $1 \mathrm{~cm}$ of the optical axis. However, such rays constitute a negligible contribution to the system's detection of light.

An isotropic, uniform, cylindrical luminescent volume is used so that variations in detected irradiance are due solely to the optical characteristics of the lens-detector system. We assume azimuthal symmetry, and so confine our simulation to the $z-\rho$ grid. The Monte Carlo data were created by generating $5 \times 10^{5}$ rays in random directions from each point in the grid. These rays were required to have a negative $z$ component of their direction vector.

The cylindrical luminescent volume is $30 \mathrm{~cm}$ in length and $20 \mathrm{~cm}$ in diameter (figure 1). At one end is a symmetric biconvex lens $5 \mathrm{~cm}$ in diameter with focal length $f=10 \mathrm{~cm}$, centred on the optical $(z)$ axis, and located at $z=0$. Grid points in the cylindrical volume are at $0.1 \mathrm{~mm}$ intervals in both the $z$ and $\rho$ directions. In the configuration we studied, a detector $1 \mathrm{~cm}$ in diameter with efficiency equal to unity is centred on the optical axis at a distance $z=-\ell$. The distance $\ell$ is varied in $1 \mathrm{~cm}$ steps.

\section{Simulation data}

Optical systems typically are described in terms of their imaging characteristics. By way of contrast, the principal characteristic we wish to explore is the collection of light from a volume, 

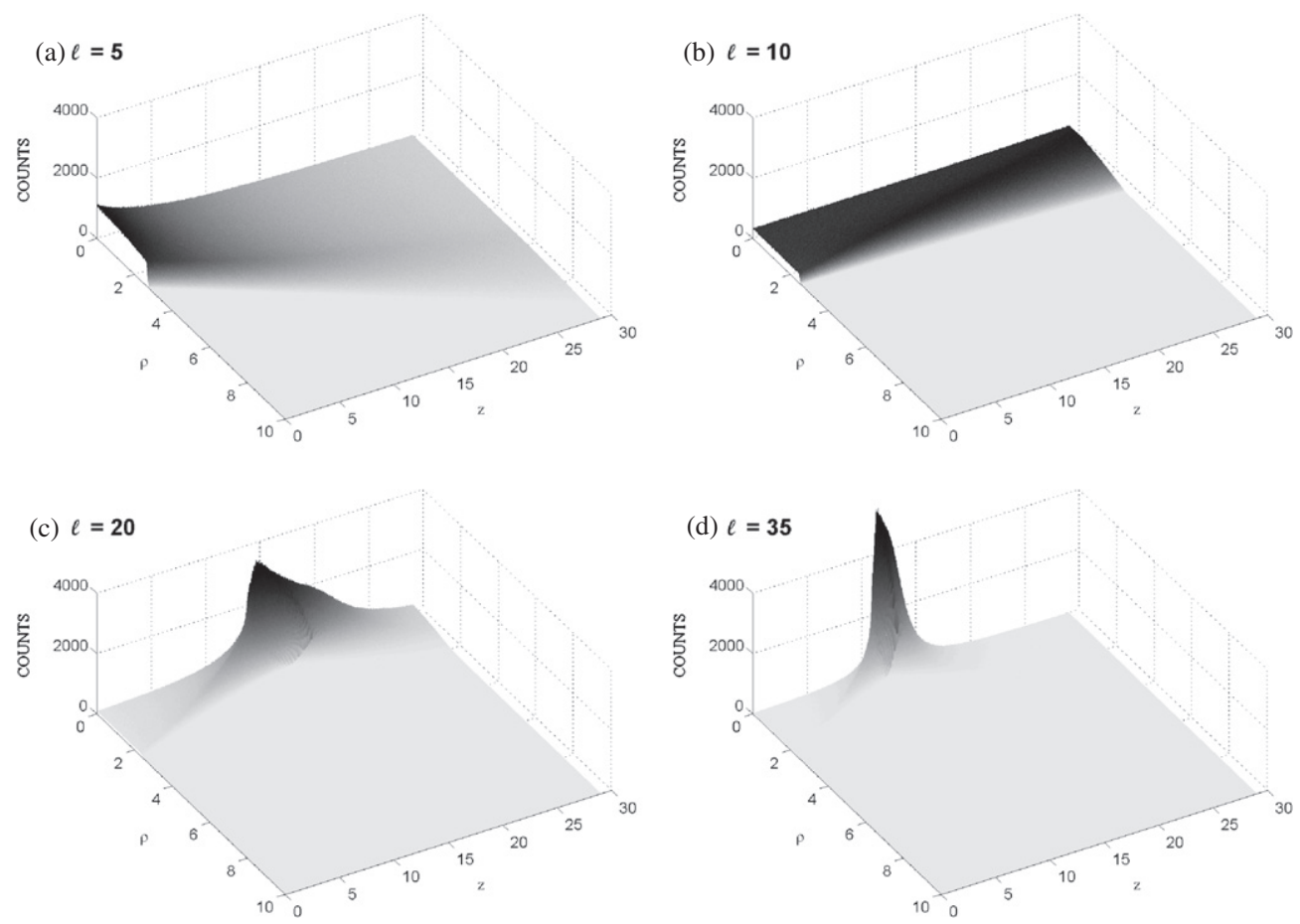

Figure 2. Number of rays detected from points in the $z-\rho$ grid for specific values of $\ell$. The grey scale represents quantities, ranging from white (smallest) to black (largest).

and we use ray tracing through a lens for this purpose. We count a ray as having been detected if it passes through the lens and crosses the bounded plane of the detector. The quantities of interest in the simulation data are the number of rays detected from each grid point, the number of grid points from which light is detected, and the locations of these grid points. We use the number of rays as a measure of the amount of light detected, and the number of grid points as a surrogate measure of the associated volume, where the radial dependence of $\mathrm{d} V$ scales as $\rho \mathrm{d} \rho$. These quantities are then used to answer the following questions: where should the detector be placed to collect the maximum irradiance, where should the detector be placed to collect the largest volume and what is the spatial distribution of the detected irradiance?

In figure 2, we provide examples of the number of rays detected as a function of $\rho$ and $z$ for various detector distances $\ell$. In figure 3 , we show a surface plot of the number of rays detected along the $z$ axis for the entire range of detector distances. As is evident from both figures 2 and 3, the amount of rays detected varies significantly depending on the location from which the rays emanate. Moreover, given that there are approximately $1.45 \times 10^{12}$ rays emitted in random directions from within the $z-\rho$ grid, only a small number of these rays hit the detector.

\section{Discussion}

When the detector is less than one focal length $(\ell<f=10 \mathrm{~cm})$ from the lens, the majority of the volume detected is also within one focal length from the lens, and the detected irradiance, or number of rays hitting the detector, decreases with increasing $\ell$ (figures 2 (a) and 3 ). When the detector is at the focal length $(\ell=f)$, light is detected from the entire length of the optical 


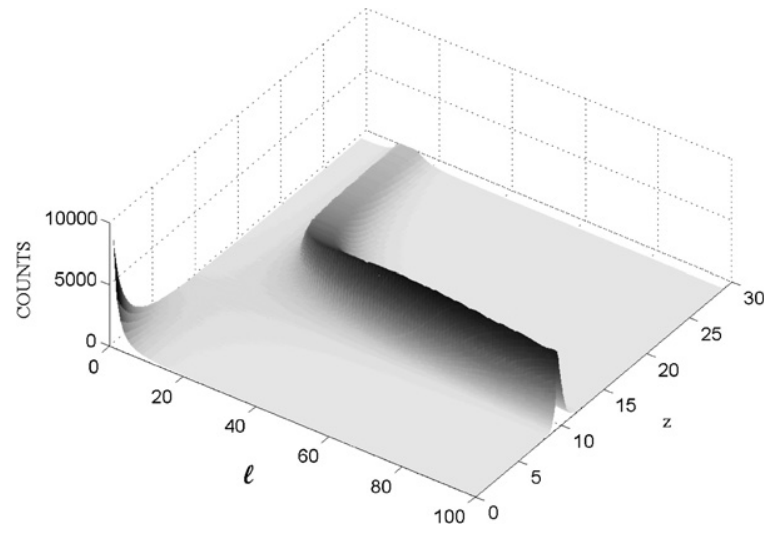

Figure 3. Number of rays detected from points on the $z$ axis for the entire range of detector distances $(\ell)$. The grey scale represents quantities, ranging from white (smallest) to black (largest).

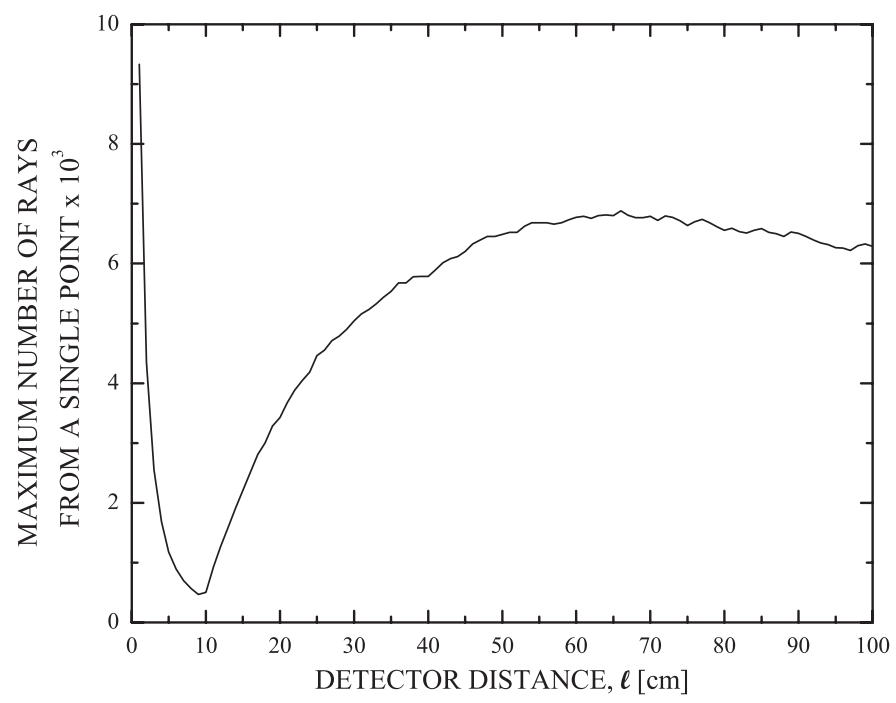

Figure 4. Maximum number of rays, $M$, seen from any single point in the $z-\rho$ grid as a function of the detector distance $\ell$.

axis (figures 2(b) and 3). For this distance, the number of rays detected per point does not vary significantly for the majority of grid points from which light is detected. Beyond the focal length $(\ell>f)$, the extent of the volume detected along the optical axis decreases with $\ell$ (figures 2(c), (d) and 3), while the maximum ray count from points along the optical axis generally increases (figure 3 ). Although figures 2 and 3 are specific to the particular geometry of the example we chose to consider, they provide a graphically simple picture of what a lens-detector combination actually 'sees'.

In figure 4 , we provide the maximum single-point ray count, $M(\ell),{ }^{2}$ for each detector distance. Figure 4 is essentially a side view of figure 3 looking along the $z$ axis. The qualitative behaviour of $M$ as a function of $\ell$ can be understood simply in terms of the maximum emission solid angle, $\Omega$, from which rays reach the detector. Corresponding values of $M$ derived from

2 In the rest of this paper we suppress the functional dependence of $M$ on $\ell$. 
the thin-lens approximation, either by ray tracing or by the analysis of the emission solid angle, are qualitatively similar. However, the thin-lens approximation produces quantities that are larger than those generated by our approximation, in some cases by as much as $30 \%$ (data not shown).

For very small $\ell, \Omega$ is almost $2 \pi$ and $M$ has a global maximum. This is similar to the effect one would have in the absence of a lens. As $\ell$ approaches $f, M$ falls to a minimum since only incident light that is approximately parallel to the optical axis intersects the detector. As $\ell$ increases beyond $f$, the $z$ coordinate of the maximum, $z_{M}$, decreases, generating increasing values of $\Omega$, and consequently increasing values of $M$. As $\ell$ increases above $\approx 70 \mathrm{~cm}$ though, this effect is overridden by the detector's finite size, resulting in a decreasing ability to intercept rays from an essentially constant $z_{M}$ (figure 3 ), and a decrease in both $\Omega$ and $M$ (figure 4). At $\ell=100, z_{M}=10.52$ and by $\ell=1000$ (not shown), $z_{M}=f$, as would be expected from the thin-lens equation. Within our statistical accuracy, $M$ corresponds to points on the $z$ axis. Specifically, the average difference between the maximum and the maximum for $\rho=0 \mathrm{~cm}$ is less than $1 \%$, and for $\ell>60 \mathrm{~cm}$, the difference is zero.

As a way to quantify the region in the cylindrical volume to which the detector is particularly sensitive, we define the 'sensitive volume', $V_{s}$, to comprise those points from which the number of rays hitting the detector is at least $M / 2$ for that detector distance. This approach roughly corresponds to using the lens focus in imaging optics to differentiate between regions in object space. In imaging optics, the aperture and the largest acceptable diameter for the circle of confusion are used to distinguish between what is 'in focus' and what is not [9]. In our data, $M$ is proportional to the maximum emission solid angle for a given detector distance. This can be translated into the equivalent of a lens aperture, and we may consider the detector as the complement to the circle of confusion.

Continuing the analogy with imaging systems, we concentrate on the system's ability to detect rays emanating from $V_{s}$ as opposed to its ability to form a focused image. From this perspective, the extent of the volume from which any rays are detected (the 'detected' volume) is analogous to the region from which the whole (focused and unfocused) image is formed. However, the sensitive volume is what the detector 'sees in focus' in our simulation and, since $V_{s}$ is a subset of the detected volume, its extent becomes the effective field of view with $V_{s}$ replacing the object as the item of interest.

In figure 5, we use semi-logarithmic plots to depict both the detected volume and $V_{s}$ as a percentage of the cylindrical volume (figure 5(a)), and the total detected irradiance compared to the irradiance from $V_{s}$ (figure 5(b)). In figure 6(a), we plot the extent of $V_{s}$ along the $z$ axis. The extent of the detected volume along the $z$ axis is not shown since for all values of $\ell$, it extends the entire length of the cylindrical volume. In figure $6(\mathrm{~b})$, we show both the extent of $V_{s}$ and the detected volume in the $\rho$ direction. The detected volume (figure 5(a)) exhibits behaviour that is qualitatively similar to that of $M$, and for similar reasons. Note that for $10 \mathrm{~cm}<\ell<20 \mathrm{~cm}$, the detected volume in the $\rho$ direction is essentially limited to the radius of the lens (figure 6(b)).

When the detector is approximately at the focal length, both $V_{s}$ and the associated irradiance are at their respective maxima. It is here that the detected rays are essentially parallel to the optical axis and consequently the $z$ coordinate of ray origin is largely unimportant. The depth of $V_{s}$ along the $z$ axis is greatest in this region. From an imaging perspective, this corresponds to an image distance equal to the focal length and an infinite object distance. Additionally, the irradiance from $V_{s}$ is high since $M$ is at a minimum here and the ray counts are similar enough for most points detected to be included in the sensitive volume.

As the detector distance increases beyond $\ell=f$, both $V_{s}$ and its irradiance decrease. Additionally, $V_{s}$ becomes more localized, as demonstrated by the contraction in the depth of 


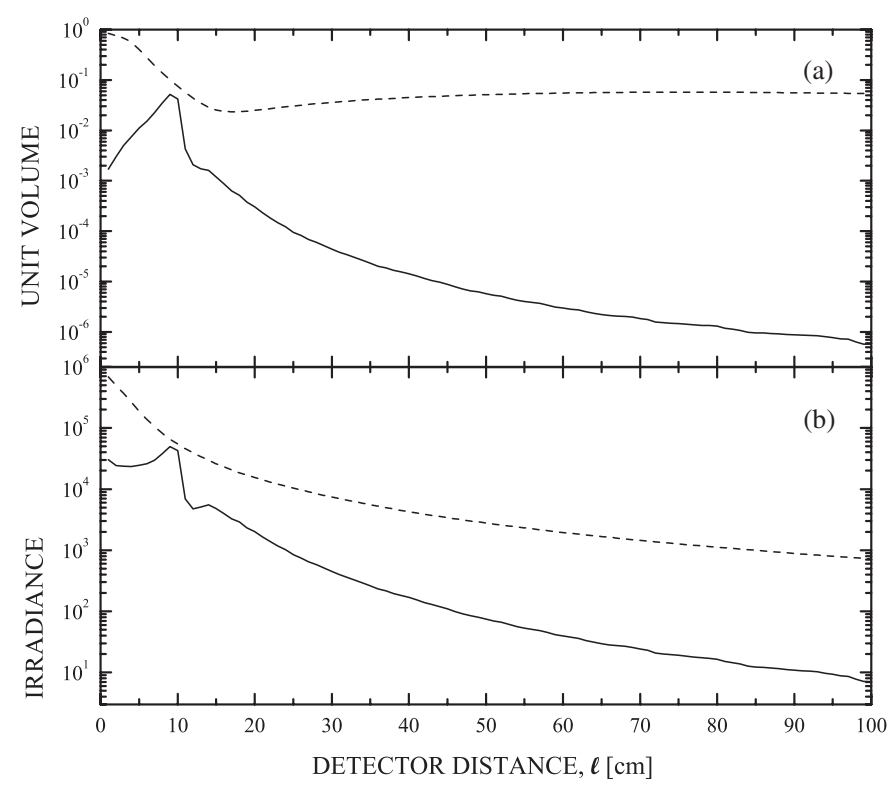

Figure 5. (a) Semi-logarithmic plots of total detected volume (dashed line) and sensitive volume (solid line), $V_{s}$, as a function of the detector distance $\ell$. The vertical axis corresponds to the fraction of the cylindrical volume occupied by these quantities; (b) semi-logarithmic plots of total detected irradiance (dashed line) and sensitive volume irradiance (solid line) as a function of the detector distance $\ell$. The vertical axis corresponds to the number of rays hitting the detector.

$V_{s}$ along the $z$ axis, and its central region moves towards the lens. The radius of $V_{s}$ is only appreciable for $\ell \lesssim 20 \mathrm{~cm}$. The sensitive volume continues to decrease relative to the detected volume until at $\ell=100 \mathrm{~cm}$ there is five orders of magnitude difference between them. The sensitive volume irradiance also continues to decrease relative to the total detected irradiance, but to a much lesser degree. At $\ell=100 \mathrm{~cm}$, there is about two orders of magnitude difference between them.

The transition from detecting an extended source to detecting a localized volume gives rise to the features at $\ell \approx 14 \mathrm{~cm}$ in $V_{s}$, its irradiance, its extent along $z$ and its extent in $\rho$. However, these features are also artefacts of the finite size of the cylindrical volume. To use imaging terminology, as the image distance increases, the object distance eventually moves close enough to the lens to appear inside the cylindrical volume. That is, for $f<\ell<14 \mathrm{~cm}$ the central region of $V_{s}$ is beyond the end $(z=30 \mathrm{~cm})$ of the cylindrical volume. By the time $\ell=14 \mathrm{~cm}$, there is enough of $V_{s}$ inside the cylinder to result in these features ${ }^{3}$. As the detector distance increases beyond $\ell>14 \mathrm{~cm}$, the angles of the rays incident on the detector tend towards zero. This is consistent with the volume from which the rays originate decreasing, and its location moving towards the focal length.

By comparing the information in figures 2-6, we see a generally inverse relationship between $M$ and both the sensitive volume and its irradiance. The extent of $V_{s}$ along the optical axis (figure 6(a)) is the effective depth of field, and $M$ corresponds to an aperture. In this context, we note that for an imaging system the depth of field increases as the aperture size

3 For a cylindrical volume $15 \mathrm{~cm}$ long, these features occur at $\ell \approx 30 \mathrm{~cm}$. When the volume is $50 \mathrm{~cm}$ long, these features do not appear. 


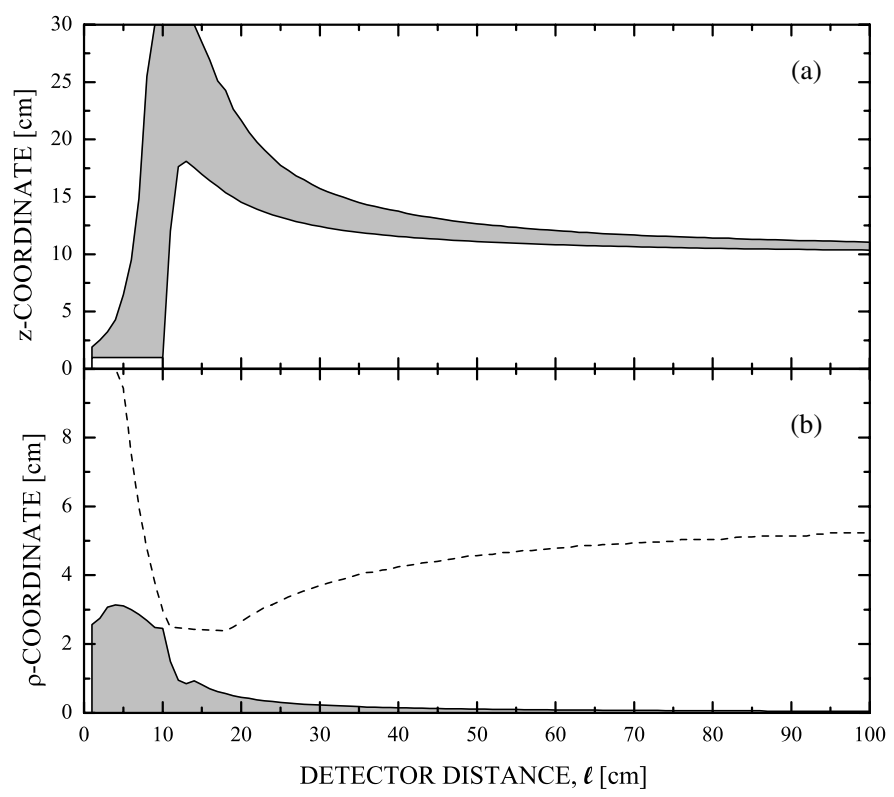

Figure 6. (a) The extent of $V_{s}$ along the $z$ axis (shaded area), or the effective depth of field. The extent of the detected volume along the $z$ axis is not shown since for all values of $\ell$ it extends the entire length of the cylindrical volume; (b) the extent of the detected volume in the $\rho$ direction (dashed line), and the extent of $V_{s}$ in the $\rho$ direction (shaded area).

decreases [9]. As a result, the inverse relationship between $M$ and the depth of $V_{s}$ is analogous to the inverse relationship between aperture size and depth of field.

The size and shape of the volume 'seen' by the lens-detector combination are a function of the detector distance. At the focal length, the optical axis of the entire cylinder is seen by the detector, and both $V_{S}$ and its irradiance are at their respective maxima. These quantities are relatively sensitive to this placement, since if the detector is more than a few centimetres away from the focal length of the lens, both $V_{s}$ and its irradiance are well below their respective maxima. Additionally, the sensitive volume is also a function of the criterion used to differentiate it from the total detected volume. For the purpose of this simulation, we somewhat arbitrarily used $M / 2$ as that criterion, with the detector assumed to have efficiency equal to unity. Specific detectors may give rise to different criteria.

\section{Summary and conclusions}

We have presented a Monte Carlo simulation as an aid to visualizing what a lens-detector combination actually detects. As ray tracing was to be an integral part of this simulation, we rejected the thin-lens approximation due to its inherent paraxial limitations, and instead used a modified approximation. We substituted the concept of a sensitive volume, $V_{s}$, for the ability to focus on an object, and used the simulation to determine how well a lens-detector configuration collects light from such a volume.

We found a number of characteristics associated with this approach to be analogous to concepts from imaging optics. The sensitive volume replaces the object, the size of the detector plays a role similar to the circle of confusion and the extent of $V_{s}$ is the effective field of view 
for the system. There is a general, inverse relationship between the maximum number of rays, $M$, and the sensitive volume, $V_{s}$, which corresponds to the inverse relationship between aperture size and depth of field.

In the configuration we studied, the preferred detector placement to maximize both the sensitive volume and its irradiance is at $\ell \approx f$. This placement results in a sensitive volume that extends the entire length of the cylindrical luminescent volume and an appreciable distance off the optical axis. As the detector distance increases beyond $\ell \approx 2 f$, the system is only able to collect light from a decreasing volume which becomes increasingly localized.

From the standpoint of determining the amount, extent and spatial distribution of what a simple optical system can detect from an extended source, these results are not surprising. Additionally, although some of these results are qualitatively similar to those that may be derived from the thin-lens approximation, they do not suffer the distortion associated with attempting to reconcile large angles of incidence with the paraxial approximation. This computational work does, however, provide the opportunity to draw conclusions not easily achievable using analytic methods, and provides students with qualitative insight into the performance of such optical systems.

\section{Acknowledgments}

This work was supported by NSF Grants PHY-0653379 and 0855629, and was completed using the University of Nebraska Holland Computing Center with the associated USCMS Tier-2 site at the University of Nebraska, Lincoln.

\section{Appendix}

Our Monte Carlo simulation is based on the following methodology. For each detector location along the negative $z$ axis (see figure 1), we generate $5 \times 10^{5}$ rays in random directions from each point in the $z-\rho$ grid $(0<z \leqslant 30 \mathrm{~cm}, 0 \leqslant \rho \leqslant 10 \mathrm{~cm})$, and require that each direction vector have a negative $z$ component. We then calculate which rays intersect the biconvex lens (radii of curvature $R=8 \mathrm{~cm}$, centred on the $z$ axis and located at $z=0$ ) and the distance $h$ from the $z$ axis at which they intersect the lens. For those rays that pass through the lens (see below), we count how many are detected.

This simulation geometry makes it possible to have angles of incidence on the lens ranging from $-\pi / 2$ to $\pi / 2$. Therefore, because of the paraxial limitations inherent in the thin-lens approximation, we adopt a modified approach to ray tracing through the lens (figure A1). This modified approximation is much simpler computationally than simulating ray propagation through a real lens.

Our modified approximation assumes a zero-thickness lens so that the distance from the axis for both entry and exit is the same, $h=h_{i}=h_{t}$ (figure A1(b)). Using the angle of incidence, $\theta_{i}$, relative to the lens normal at distance $h$, we use Snell's law to compute the refracted transmission angle in the lens, assuming that the incident and transmitted indices of refraction are $n_{i}=1$ and $n_{t}=1.4$, respectively. The transmitted ray angle is then used to compute the internal angle of incidence as the ray emerges from the downstream surface of the lens. Using Snell's law a second time, we compute the emerging transmission angle, $\theta_{t}$ (figures A1(c) and (d)). The transmission angle, $\theta_{t}$, is given by

$$
\sin \theta_{t}=n_{t} \sin (|\gamma|)
$$


(a)

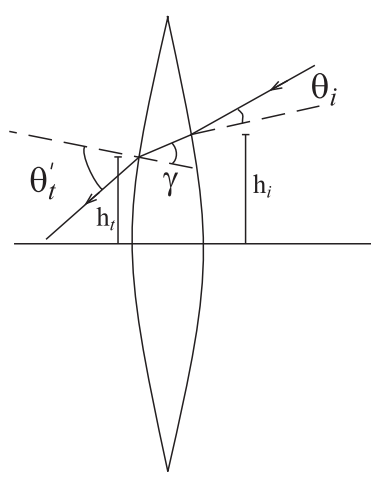

(c)

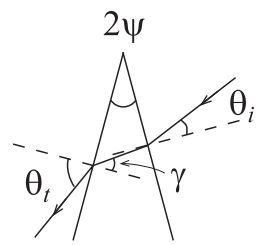

(b)

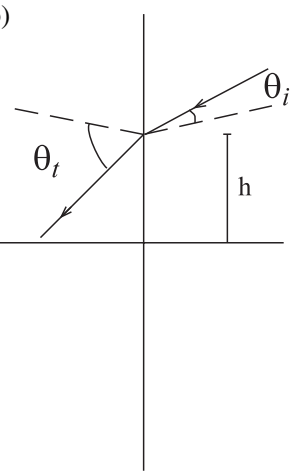

(d)

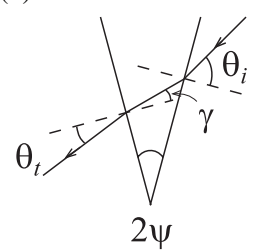

Figure A1. Comparison of real-lens ray tracing to our modified approximation (see the text). (a) Ray tracing through a real lens. Note that the distances from the optical axis for rays entering and leaving the lens $\left(h_{i}\right.$ and $h_{t}$, respectively) are in general different. The incident angle is $\theta_{i}$ and the transmission angle is $\theta_{t}^{\prime}$. (b) Ray tracing through a lens using our modified (non-thin lens) approximation. Since there is no optical path length through the lens, $h=h_{i}=h_{t}$. The angle of incidence $\theta_{i}$ is the same as in the real-lens case, but the angle of transmission is in general different, $\theta_{t} \neq \theta_{t}^{\prime}$. (c) and (d) Geometrical elements corresponding to equations (A.2) and (A.3), respectively. Since $h=h_{i}=h_{t}$, these triangles reflect how our approximation computes $\theta_{t}$.

where $\gamma$, the internal angle of incidence, is given by

$$
\begin{aligned}
& \gamma=2 \psi+\sin ^{-1}\left(\frac{\sin \theta_{i}}{n_{t}}\right) \text { or } \\
& \gamma=2 \psi-\sin ^{-1}\left(\frac{\sin \theta_{i}}{n_{t}}\right)
\end{aligned}
$$

depending on the relationship between $\theta_{i}$ and the normal to the lens surface (equation (A.2), figure A1(c); equation (A.3), figure A1(d)). The angle $\psi$ between the vertical and the tangent to the lens surface at the point of intersection is given by

$$
\sin \psi=\frac{h}{R}
$$

Based upon the direction of the ray and its location as it leaves the lens, we determine if it intersects the detector. For each ray that intersects the detector, we increase the counter associated with its point of origin in the $z-\rho$ grid. Once all $5 \times 10^{5}$ random-direction rays have been generated and tested for each point in the $z-\rho$ grid, the detector location is moved to its next position and the simulation repeats.

While this modified approximation is better than the paraxial thin-lens approximation, there is an approximation error. As a measure of this error, we show in figure A2 the difference 


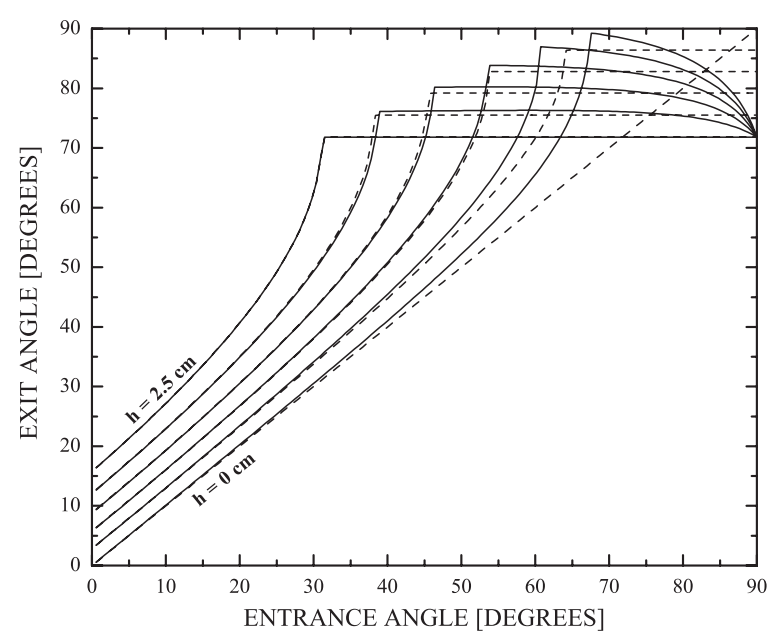

Figure A2. Exit angles relative to the optical axis for a real lens (solid lines; see figure A1(a)) and our modified approximation (dashed lines; see figure A1(b)) at distances $h=0-2.5 \mathrm{~cm}$ (in $0.5 \mathrm{~cm}$ increments) from the optical axis. The horizontal axis represents entrance angles relative to the optical axis. The cusps in both sets of exit angles are due to the onset of total internal reflection. Note that for $h=2.5 \mathrm{~cm}$, approximation exit angles are indistinguishable from real-lens exit angles.

between the exit angles ${ }^{4}$ relative to the optical axis for both our modified approximation and for a real lens. These exit angles are plotted as a function of the entrance angle, also relative to the optical axis, when passing through the lens at various distances from the optical axis. The cusps that appear in both sets of exit angles are due to the onset of total internal reflection. We compare our modified approximation to a real lens of diameter $5 \mathrm{~cm}$ with $R=8 \mathrm{~cm}$ and width $d=0.8 \mathrm{~cm}$ for distances of $h=0-2.5 \mathrm{~cm}$ (in $0.5 \mathrm{~cm}$ increments) from the optical axis. The difference between the approximated and actual exit angles generally increases with the angle of the incoming ray. We note, however, that this difference does not exceed $10^{\circ}$ until the entrance angle increases beyond $60^{\circ}$ and $h \leqslant 1 \mathrm{~cm}$. In general, for an entrance angle of $60^{\circ}$ or less, the error is no more than $5 \%$ so that, even when the detector is in the immediate vicinity of the lens, the effect of the error on the number of rays intersecting the detector is negligible.

\section{References}

[1] Berry H G 1977 Beam-foil spectroscopy Rep. Prog. Phys. 40 155-217

[2] Maseberg J W and Gay T J 2009 Spin torque on molecular rotation induced by polarized electrons Phys. Rev. A 79022705

[3] Stopper U, Lindner P and Schumacher U 2007 Three-dimensional analysis of microwave generated plasmas with extended planar laser-induced fluorescence Rev. Sci. Instrum. 78043508

[4] Boffard J B, Jung R O, Lin C C and Wendt A E 2010 Optical emission measurements of electron energy distributions in low-pressure argon inductively coupled plasmas Plasma Sources Sci. Technol. 19065001

[5] Gu M 2000 Advanced Optical Imaging Theory (Berlin: Springer) p 79

[6] Winston R, Minano J C and Benitez P 2005 Nonimaging Optics (Boston, MA: Elsevier) p 75

[7] Born M and Wolf E 1975 Principles of Optics 5th edn (New York: Pergamon) p 440

[8] Hecht E 2002 Optics 4th edn (New York: Addison-Wesley) pp 163-5

[9] Pedrotti F L, Pedrotti L S and Pedrotti L M 2007 Introduction to Optics 3rd edn ((Englewood Cliffs, NJ: Prentice-Hall) pp $72-3$

4 We use the terms 'exit' and 'entrance' angles to distinguish angles measured relative to the optical axis from transmission and incident angles, which are measured relative to the lens-surface normal. 